山्山FFRANÇAISE

$>\mathrm{DE}$

님 PÉDAGOGIE

\section{Revue française de pédagogie}

Recherches en éducation

156 | juillet-septembre 2006

Les espaces locaux d'interdépendance entre

établissements : une comparaison européenne

\title{
FASSA Farinaz. Société en mutation, école en transformation : le récit des ordinateurs
}

Lausanne : Éd. Payot Lausanne, 2005. - 448 p. (Hic \& Nunc)

Georges-Louis Baron

\section{OpenEdition}

\section{Journals}

\section{Édition électronique}

URL : http://journals.openedition.org/rfp/658

DOI : $10.4000 /$ rfp.658

ISSN : 2105-2913

Éditeur

ENS Éditions

Édition imprimée

Date de publication : 1 juillet 2006

Pagination : 181-182

ISBN : 978-2-7342-1060-3

ISSN : 0556-7807

Référence électronique

Georges-Louis Baron, « FASSA Farinaz. Société en mutation, école en transformation : le récit des ordinateurs ", Revue française de pédagogie [En ligne], 156 | juillet-septembre 2006, mis en ligne le 24 septembre 2010, consulté le 25 septembre 2020. URL : http://journals.openedition.org/rfp/658 ; DOI : https://doi.org/10.4000/rfp.658

Ce document a été généré automatiquement le 25 septembre 2020

(C) tous droits réservés 


\title{
FASSA Farinaz. Société en mutation, école en transformation : le récit des ordinateurs
}

\author{
Lausanne : Éd. Payot Lausanne, 2005. - 448 p. (Hic \& Nunc)
}

Georges-Louis Baron

\section{RÉFÉRENCE}

FASSA Farinaz. Société en mutation, école en transformation : le récit des ordinateurs.

Lausanne : Éd. Payot Lausanne, 2005. - 448 p. (Hic \& Nunc)

1 Ce livre, issu d'une thèse de doctorat soutenue à l'université de Lausanne en 2004 sous la direction de Gerald Berthoud, s'intéresse à la prise en compte par l'école de l'informatique puis des technologies de l'information et de la communication dans le canton de Vaud.

Dans la première partie du livre, l'auteure donne des indications sur sa propre implication: à l'origine enseignante d'histoire dans l'enseignement post-obligatoire, elle a été pionnière de l'informatique à l'école et a même développé, à la fin des années 1980, des connaissances assez poussées dans ce domaine. Mais ses expériences ont été relativement frustrantes. Elle a alors adopté une démarche de recherche relativement critique. Dans ce cadre, elle a analysé, d'un point de vue ethnologique, la question des ordinateurs à l'école en s'intéressant en particulier aux enjeux de pouvoir. Ses investigations ont été menées depuis différentes positions institutionnelles dont celle de chargé d'étude du département de la formation et de la jeunesse du canton de Vaud.

Elle indique dans le livre avoir suivi la "piste» des ordinateurs pour chercher à comprendre "comment l'école et ses différents acteurs professionnels envisagent aujourd'hui la culture qu'il leur appartient de transmettre à leurs élèves» (p. 81). Faisant, de manière pertinente, la distinction entre informatique dans, pour et à l'école, elle s'est principalement fondée sur une enquête auprès de décideurs de l'introduction 
puis de la diffusion de l'informatique dans l'école vaudoise. Elle a également utilisé pour son argumentation deux ensembles de sources. Le premier comprend des textes qualifiés de " pragmatiques ", caractérisés par la proposition d'« un passage immédiat à l'action en lui fournissant un modèle ou un cadre qu'il ne s'agit que peu de transformer" (p. 99). Pour une autre part, il s'agit de textes à caractère " programmatique ", issus de l'institution.

Ce choix méthodologique informe effectivement sur ce qui relève de l'incitation à l'usage d'ordinateurs. Il a en revanche un coût: focalisant l'attention sur les représentations dominantes, il conduit à sous-estimer les initiatives lancées par les enseignants. En Suisse comme ailleurs, un ensemble de pédagogues "en recherche", alliés à des chercheurs, ont conduit une réflexion relativement à la transmission de nouveaux savoirs et au développement de nouvelles activités instrumentées d'apprentissage. Ils ont produit des textes et des artefacts dont il est possible de penser qu'ils ont, comme en France, exercé une influence sensible sur la diffusion des représentations de ce que peut réellement représenter l'informatique à l'école.

5 La deuxième partie expose d'abord une très intéressante analyse des réformes mises en œuvre depuis une vingtaine d'années dans le canton de Vaud. Puis elle entreprend une narration problématisée de la prise en compte des ordinateurs dans le système scolaire en mettant en relation ce qui s'y est passé avec les initiatives prises au niveau de la Confédération helvétique ainsi que, plus largement, avec les opérations menées dans d'autres pays. Elle relève la proximité entre ce que l'on trouve dans le canton de Vaud et les visions d'organisations internationales comme l'OCDE, qui sont en phase avec les idées de capitalisme en réseau tels que les décrivent Boltanski et Chiapello.

6 Le texte nous a semblé assez convaincant, même si la question de la prise en compte des savoirs liés à l'informatique et, plus largement, aux technologies de l'information et de la communication était abordée de manière un peu décevante. De nombreux travaux ont été menés sur ces questions en Suisse, auxquels le texte ne fait pas référence. De manière surprenante, les travaux classiques de Larry Cuban sur les États-Unis ne sont pas non plus cités.

7 La troisième partie (les TIC entre impensé et inconscient) revient sur les tensions entre ce qui relève de la technique, des valeurs traditionnelles de l'école et des nouvelles orientations politiques sensibles à l'influence d'une image de modernité.

8 Le livre s'achève sur une série de réflexions critiques sur l'alliance possible entre politiques néo-libérales, société de l'information et technologies (ces dernières servant d'arguments aux premières). D'après l'auteur, cette alliance pourrait conduire à un risque pour l'avenir, celui d'une "société de l'information » ayant un idéal éducatif «d'apprenance permanente» conduisant en fait à la «fragilisation» des individus. L'argumentation, qui semble viser à mettre en garde contre les risques d'un rapport subjugué à la technique, ne manque pas de cohérence. Elle revendique un cadre théorique bien précisé, mais elle a un caractère largement spéculatif et paraît un peu déconnectée du recueil de données empiriques.

9 Au total, l'ouvrage, qui n'est pas d'une lecture toujours facile du fait de la présence d'une multitude de notes de bas de page et de références à des écrits théoriques (tribut probablement payé au genre de la thèse), aborde de manière cohérente un problème intéressant. Limité par une approche mettant en valeur les points de vue de responsables de la mise en œuvre de politiques scolaires, il a le mérite d'éclairer le 
processus de prise en compte de l'informatique dans le système scolaire vaudois et de le mettre en perspective avec ce qui s'est passé dans d'autres pays industrialisés.

\section{AUTEURS}

\section{GEORGES-LOUIS BARON}

Université Paris 5-René Descartes 